\title{
Tenofovir disoproxil fumarate monotherapy for nucleos(t)ide analogue-naïve and nucleos(t)ide analogue-experienced chronic hepatitis B patients
}

\author{
Sang Kyung Jung', Kyung-Ah Kim', So Young Ha', Hyun Kyo Lee', Young Doo Kim', Bu Hyun Lee', Woo Hyun Paik', \\ Jong Wook Kim', Won Ki Bae', Nam-Hoon Kim', June Sung Lee', and Yoon Jung Jwa² \\ 'Department of Internal Medicine, Ilsan Paik Hospital, Inje University College of Medicine, Goyang; ${ }^{2}$ Health promotion center Ilsan Paik \\ Hospital, Inje University College of Medicine, Goyang, Korea
}

Background/Aims: This study investigated the antiviral effects of tenofovir disoproxil fumarate (TDF) monotherapy in nucleos(t)ide analogue (NA)-naive and NA-experienced chronic hepatitis B (CHB) patients.

Methods: CHB patients treated with TDF monotherapy (300 mg/day) for $\geq 12$ weeks between December 2012 and July 2014 at a single center were retrospectively enrolled. Clinical, biochemical, and virological parameters were assessed every 12 weeks.

Results: In total, 136 patients (median age 49 years, 96 males, $94 \mathrm{HBeAg}$ positive, and 51 with liver cirrhosis) were included. Sixty-two patients were nucleos(t)ide (NA)-naïve, and 74 patients had prior NA therapy (NA-exp group), and 31 patients in the NA-exp group had lamivudine (LAM)-resistance (LAM-R group). The baseline serum hepatitis B virus (HBV) DNA level was $4.9 \pm 2.3 \log \mathrm{IU} / \mathrm{mL}$ (mean $\pm \mathrm{SD}$ ), and was higher in the NA-naïve group than in the NA-exp and LAM-R groups $(5.9 \pm 2.0 \log \mathrm{IU} / \mathrm{mL}$ vs $3.9 \pm 2.0 \log \mathrm{IU} / \mathrm{mL}$ vs $4.2 \pm 1.7 \log \mathrm{IU} / \mathrm{mL}, P<0.01)$. The complete virological response (CVR) rate at week 48 in the NA-naïve group (71.4\%) did not differ significantly from those in the NA-exp (71.3\%) and LAM-R (66.1\%) groups. In multivariate analysis, baseline serum HBV DNA was the only predictive factor for a CVR at week 48 (hazard ratio, 0.809; 95\% confidence interval, 0.729-0.898), while the CVR rate did not differ with the NA experience.

Conclusions: TDF monotherapy was effective for CHB treatment irrespective of prior NA treatment or LAM resistance. Baseline serum HBV DNA was the independent predictive factor for a CVR. (Clin Mol Hepatol 2015;21:41-48)

Keywords: Chronic Hepatitis B; Tenofovir; Nucleos(t)ide analogue-experienced; Lamivudine-resistant

\section{INTRODUCTION}

Chronic hepatitis B virus (HBV) infection is a major cause of cirrhosis and hepatocellular carcinoma (HCC) worldwide. The primary treatment goal of chronic HBV infection is to prevent the development of liver cirrhosis and HCC. The natural course of chronic HBV infection is highly diverse at the individual level, ranging from inactive carriers to end-stage liver disease or HCC. While many viral

\section{Abbreviations:}

ADV, adefovir; ALT, alanine aminotransferase; $C H B$, chronic hepatitis $B ; C V R$, complete virological response; $H B V$, hepatitis B virus; LAM, lamivudine; NA, nucleos(t)ide analogue; PVR, partial virological response; TDF, tenofovir disoproxil fumarate

\author{
Corresponding author : Kyung-Ah Kim \\ Department of Internal Medicine, Ilsan Paik Hospital, Inje University \\ College of Medicine, 170 Juhwaro, Ilsanseogu, Goyang 411-706, Korea. \\ Tel: +82-31-910-7200, Fax: +82-31-910-7219 \\ E-mail: kakim@paik.ac.kr
}


and host factors can affect this course, the serum HBV DNA level is one of the most important factors affecting prognosis., ${ }^{2,3}$ The long-term suppression of HBV replication is therefore an important goal in the effective treatment of chronic HBV infection. ${ }^{4,5}$

Tenofovir disoproxil fumarate (TDF) is an oral prodrug of tenofovir, an acyclic nucleoside phosphonate analogue of adenosine 5 '-monophosphate, with an excellent safety profile and potent anti-HBV efficacy in adults. ${ }^{6,7}$ Up to 6 years of treatment with TDF, long term suppression of HBV can lead to regression of fibrosis and cirrhosis without emergence of resistance to TDF in nucleos(t) ide analogue (NA)-naïve patients. ${ }^{8,9}$ Apart from the demonstrated efficacy in naïve chronic hepatitis $B$ patients, it has been recently reported to be effective in patients who previously failed lamivudine (LAM) and/or adefovir (ADV). ${ }^{10-12}$ However, most of these studies were conducted in region where genotype $A$ or $D$ is dominant, whereas most Korean patients are infected with genotype $C$ $\mathrm{HBV}^{13}{ }^{13}$ TDF was approved for the treatment of CHB in December 2012 in Korea. One-year data showed TDF monotherapy is effective and safe in NA-naïve Korean patients. ${ }^{14}$ However, there is few data on antiviral efficacy in LAM-experienced or LAM-resistant Korean patients.

We aimed to evaluate the antiviral efficacy of TDF monotherapy in both NA-naïve and NA-experienced patients and clarify TDF monotherapy is also effective in LAM-resistant patients.

\section{METHODS}

\section{Patients}

Patients with chronic HBV infection who were treated with TDF monotherapy (300 mg/day) for at least 12 weeks between December 2012 and July 2014 at a single center were consecutively enrolled. Patients with coinfection with hepatitis C virus or HIV and patients who have experienced multi-drug resistance and were not indicated for TDF monotherapy were excluded. The study protocol was approved by the institutional review board of Ilsan Paik Hospital, Inje University College of Medicine, Goyang, Korea.

\section{Data collection}

History of previous antiviral treatment, antiviral drug resistance, presence of cirrhosis and adherence to medication were collected. $H B e A g, H B e A b$, serum alanine aminotransferase (ALT), and serum HBV DNA were also collected at baseline and every 12 weeks. Vi- ral mutation tests were performed at baseline in NA-experienced patients and in patients with virological breakthrough (VBT).

\section{Laboratory assay}

Serologic markers of HBV were tested using commercially available electrochemiluminescence immunoassay (ECLIA, Roche Diagnostics, Branchburg, NJ). Serum HBV DNA levels were measured by real-time PCR using The COBAS ${ }^{\circledR}$ Amplicor assay (Roche Diagnostics, limit of detection $20 \mathrm{lU} / \mathrm{mL}$ ). Genotypic analysis was done by the restriction fragment mass polymorphism (RFMP) method.

\section{Definition}

A complete virological response (CVR) is defined as a decrease in serum HBV DNA to an undetectable level as determined by realtime $P C R$ assay $(\leq 20 \mathrm{IU} / \mathrm{mL}$ ). A partial virological response (PVR) is defined as a decrease in HBV DNA of more than $2 \mathrm{log} \mathrm{IU} / \mathrm{mL}$ but with detectable serum HBV DNA at week 48 for entecavir (ETV), ADV and TDF, and at week 24 for LAM and telbivudine (LdT). ${ }^{15}$ VBT is defined as a confirmed increase in HBV DNA level of more than $1 \log \mathrm{IU} / \mathrm{mL}$ compared to the nadir HBV level achieved during therapy. Biochemical response is defined as a normalization of ALT ( $\leq 40 \mathrm{IU} / \mathrm{L})$. Diagnosis of liver cirrhosis was based on the histological findings or radiological findings together with clinical features indicative of portal hypertension, such as thrombocytopenia (platelet count $<100,000 / \mathrm{mm}^{3}$ ), gastroesophageal varices, or ascites.

\section{Statistical analysis}

Continuous variables were summarized as the median (interquartile range) or mean \pm SD. Serum HBV DNA levels were logarithmically transformed for analysis. Continuous variables were compared using the Student's $t$-test, ANOVA, nonparametric Mann-Whitney or Kruskal-Wallis test as appropriate and categorical variables were compared using chi-square test. CVR rate was calculated with Kaplan-Meier's curve and compared with the log rank test. A Cox proportional hazard model including variables with $P$-values $\leq 0.1$ in univariate analysis and clinically relevant factors was used to identify predictive factors independently associated with the time to a CVR. $P$-value $<0.05$ was considered statistically significant. All statistical analyses were performed using SPSS version 21.0 (SPSS Inc., Chicago, IL, USA). 


\section{RESULTS}

\section{Baseline characteristics of patients}

From November 2012 to July 2014, a total of 212 patients were treated with TDF monotherapy. Among those, 18 patients were excluded for multidrug resistance, and 58 patients were excluded for follow-up duration $<3$ months. A total of 136 patients (male $96(70.6 \%)$, median age 49$)$ were finally included in this study. Baseline characteristics of patients were summarized in Table 1.

Ninety-four patients (69.1\%) were $\mathrm{HBeAg}$-positive and 51 patients $(37.5 \%)$ had liver cirrhosis. Median follow-up duration was 56 weeks. The mean baseline serum HBV DNA level was $4.9 \pm 2.3$ $\log \mathrm{IU} / \mathrm{mL}$ and the mean ALT level was 155 $252 \mathrm{IU} / \mathrm{L}$.

Seventy-four patients had experienced prior NA therapy (NA-exp group). Among those, 31 patients had documented antiviral resistance to lamivudine (LAM-R group). Twenty-eight patients have not been tested for genotypic mutation. The reasons for medication change to TDF in NA-experienced patients were PVR $(n=45)$, withdrawal hepatitis after discontinuation of prior NA therapy $(n=13)$, virological breakthrough $(n=10)$, emergence of antiviral resistance $(n=2)$, myopathy $(n=2)$ and pregnancy $(n=2)$. Mean duration of washout period in patients with withdrawal hepatitis was 49.7 weeks. Prior history of NA treatment and types of antiviral resistance were summarized in Table 2. Baseline serum HBV DNA was significantly higher in NA-naïve than NA-exp group (5.9 $\pm 2.0 \mathrm{log} \mathrm{IU} / \mathrm{mL}$ vs. $3.9 \pm 2$. $\log \mathrm{IU} / \mathrm{mL}, P<0.01$ ). Baseline serum ALT was also significantly higher in NA-naive patients than in NAexperienced patients.

Table 1. Baseline characteristics of the patients

\begin{tabular}{|c|c|c|c|c|}
\hline & All patients $(n=136)$ & NA-naïve $(n=62)$ & $N A-\exp (n=74)$ & LAM-R ( $n=31)$ \\
\hline Age, years ${ }^{*}$ & $49(41-58)$ & $47(21-70)$ & $52(23-81)$ & $55(45-59)$ \\
\hline Male sex, n (\%) & $96(70.6 \%)$ & $48(77.4 \%)$ & 48 (64.9\%) & $22(71.0 \%)$ \\
\hline Liver cirrhosis, n (\%) & $51(37.5 \%)$ & $24(38.7 \%)$ & $27(36.5 \%)$ & $14(45.2 \%)$ \\
\hline Treatment duration (weeks) & $56.6(41.7-71.0)$ & $51.5(12-83)$ & $60.7(19-81)$ & $69.9(46.9-78.4)$ \\
\hline HBeAg positivity, n (\%) & $94(69.1 \%)$ & $40(64.5 \%)$ & $54(73.0 \%)$ & $25(80.6 \%)$ \\
\hline HBV DNA $(\log I U / m L)^{\dagger}$ & $4.9 \pm 2.3$ & $5.9 \pm 2.0$ & $3.9 \pm 2.0^{\ddagger}$ & $4.2 \pm 1.7^{\ddagger}$ \\
\hline $\operatorname{ALT}(I U / L)^{\dagger}$ & $155.4 \pm 252.4$ & $221.8 \pm 292.8$ & $100.1 \pm 196.5^{\ddagger}$ & $52.5 \pm 67.9^{\ddagger}$ \\
\hline Total bilirubin $(\mathrm{mg} / \mathrm{dL})^{\dagger}$ & $1.4 \pm 2.3$ & $1.8 \pm 3.3$ & $1.1 \pm 0.7$ & $1.1 \pm 0.8$ \\
\hline Creatinine $(\mathrm{mg} / \mathrm{dL})^{\dagger}$ & $0.95 \pm 0.41$ & $0.97 \pm 0.57$ & $0.92 \pm 0.15$ & $0.9 \pm 0.2$ \\
\hline eGFR $\left(\mathrm{mL} / \mathrm{min} / 1.73 \mathrm{~m}^{2}\right)^{\dagger}$ & $81.5 \pm 17.2$ & $84.5 \pm 20.9$ & $78.9 \pm 12.6$ & $78.2 \pm 13.1$ \\
\hline
\end{tabular}

ALT, alanine aminotransferase; eGFR, estimated glomerular filtration rate; HBeAg, hepatitis B e antigen; HBV, hepatitis B virus; LAM-R, lamivudine resistant; $N A$, nucleos(t)ide analogue.

*Median (range).

${ }^{\dagger}$ Mean \pm standard deviation.

‡Significantly different as compared with NA-naïve group.

Table 2. Patterns of prior antiviral treatment and genotypic resistance analysis in NA-exp patients

\begin{tabular}{|c|c|c|c|c|c|c|c|c|}
\hline \multirow[b]{2}{*}{ Previous treatment } & \multirow[b]{2}{*}{$n$} & \multirow{2}{*}{$\begin{array}{l}\text { Treatment duration in } \\
\text { mo, median (range) }\end{array}$} & \multicolumn{6}{|c|}{ LAM resistance Variants } \\
\hline & & & Total & M204I & $\begin{array}{l}\text { M204I + } \\
\text { L180M }\end{array}$ & $\begin{array}{l}\mathrm{M} 204 \mathrm{~V} / \mathrm{I}+ \\
\mathrm{L} 180 \mathrm{M}\end{array}$ & $\begin{array}{l}\text { M204V + } \\
\text { L180M }\end{array}$ & Unclassified \\
\hline LAM or clevudine or LdT mono & 16 & $27(2-70)$ & 3 & 2 & 1 & 0 & 0 & 0 \\
\hline ETV $0.5 \mathrm{mg}$ & 25 & $23(3-60)$ & 1 & 0 & 0 & 0 & 1 & 0 \\
\hline $\begin{array}{l}\text { Sequential LAM or clevudine } \\
\text { or LdT and ADV }\end{array}$ & 11 & $26(4-57)$ & 7 & 3 & 3 & 0 & 0 & 1 \\
\hline $\begin{array}{l}\text { Add on combination LAM or } \\
\text { clevudine or LdT with ADV }\end{array}$ & 8 & $20(3-44)$ & 7 & 1 & 3 & 1 & 2 & 0 \\
\hline $\begin{array}{l}\text { Sequential LAM or clevudine } \\
\text { or LdT and ETV 1.0mg }\end{array}$ & 14 & $30(4-62)$ & 13 & 4 & 2 & 4 & 3 & 0 \\
\hline
\end{tabular}

Mo, month; LAM, lamivudine; LAM-R, lamivudine resistance; LdT, telbivudine; ADV, adefovir; ETV, entecavir. 
Table 3. Univariate and multivariate Cox proportional hazards analyses to identify factors associated with a complete virological response

\begin{tabular}{|c|c|c|c|c|c|c|}
\hline \multirow{2}{*}{ Baseline parameter } & \multicolumn{3}{|c|}{ Univariate analysis ${ }^{*}$} & \multicolumn{3}{|c|}{ Multivariate analysis ${ }^{*}$} \\
\hline & HR & $95 \% \mathrm{Cl}$ & $P$-value & HR & $95 \% \mathrm{Cl}$ & $P$-value \\
\hline Age, years $^{\dagger}$ & 1.007 & $0.990-1.024$ & 0.416 & & & \\
\hline Male sex & 1.112 & $0.888-1.391$ & 0.354 & & & \\
\hline Baseline HBV DNA, log IU/mL $\mathrm{mL}^{\dagger}$ & 0.830 & $0.757-0.911$ & $<0.001$ & 0.809 & $0.729-0.898$ & $<0.001$ \\
\hline Baseline ALT, IU/L & 0.999 & $0.998-1.001$ & 0.328 & & & \\
\hline NA-naïve vs NA-exp & 1.125 & $0.708-1.125$ & 0.619 & 1.441 & $0.923-2.250$ & 0.175 \\
\hline NA-naïve vs LMV-R & 1.116 & $0.657-1.896$ & 0.684 & & & \\
\hline Liver cirrhosis & 0.779 & $0.511-1.189$ & 0.247 & & & \\
\hline HBeAg negativity & 1.685 & $1.096-2.590$ & 0.017 & 1.200 & $0.963-1.496$ & 0.104 \\
\hline
\end{tabular}

$H R$, hazard ratio; $\mathrm{Cl}$, confidence interval; ALT, alanine aminotransferase; NA, nucleos(t)ide analogue; HBeAg, hepatitis B e antigen; HBV, hepatitis B virus; LAM, lamivudine; LAM- $R$, lamivudine resistance; ADV, adefovir.

" $P$-values and hazard ratios are calculated by Cox proportional hazard model.

${ }^{\dagger}$ Analyzed as a continuous variable.

\section{Virological and biochemical responses to TDF monotherapy}

Mean reduction of serum HBV DNA was $3.03 \mathrm{log} \mathrm{IU} / \mathrm{mL}$ at week 24 and $3.33 \mathrm{log} \mathrm{IU} / \mathrm{mL}$ at week 48 respectively. Reduction of serum HBV DNA was significantly higher in NA-naïve group than NA-Exp group both at week 24 (4.03 vs. $2.30 \log \mathrm{IU} / \mathrm{mL}, P<0.01$ ) and at week 48 (4.28 vs. $2.46 \mathrm{log} \mathrm{IU} / \mathrm{mL}, P<0.01$ ) (Fig. 1). However, there was no significant difference of the serum HBV DNA reduction between NA-naive and NA-Exp group who had washout periods for previous NA therapy and similar baseline serum HBV DNA with NA-naive patients at week 24 (4.04 vs. $4.59 \mathrm{log} \mathrm{IU} / \mathrm{mL}$, $P=0.61$ ) and week 48 (4.30 vs. $4.64 \log \mathrm{IU} / \mathrm{mL}, P=0.82$ ).

CVR was achieved in $47.1 \%$ at week 24 and $71.5 \%$ at week 48 respectively. CVR rate at week 48 in NA-naïve group (71.7\%) was not significantly different from NA-exp group ( $n=74,71.3 \%$ ) (Fig. 2A). When NA-naïve group was compared with and LMV- $R(n=31$, $66.1 \%)$, there was no significant difference of CVR between two groups. There was no difference of CVR according to types of NA (NA naive $71.7 \%$ vs. patients with exposure to LMV only $74.1 \%$ vs. patients with exposure to ETV $68.9 \%$, patients with exposure to ADV $72.9 \%$ ) either. When VR was defined as $<60 \mathrm{IU} / \mathrm{mL}$, VR at week 48 was $88.1 \%$ in NA-naïve patients, $88.1 \%$ in NA-exp group and $87.9 \%$ in LAM-R group respectively. CVR rate at week 48 was significantly higher in HBeAg-negative (87.6\%) than HBeAg positive patients (75\%) (Fig. 2B). Patients with baseline serum HBV DNA $<4.3 \log \mathrm{IU} / \mathrm{mL}$ achieved significantly higher CVR (87.8\%) than those with baseline serum HBV DNA $\geq 4.3 \log \mathrm{IU} / \mathrm{mL}$ (59.5\%) (Fig. 2C). To compensate the difference of baseline serum HBV

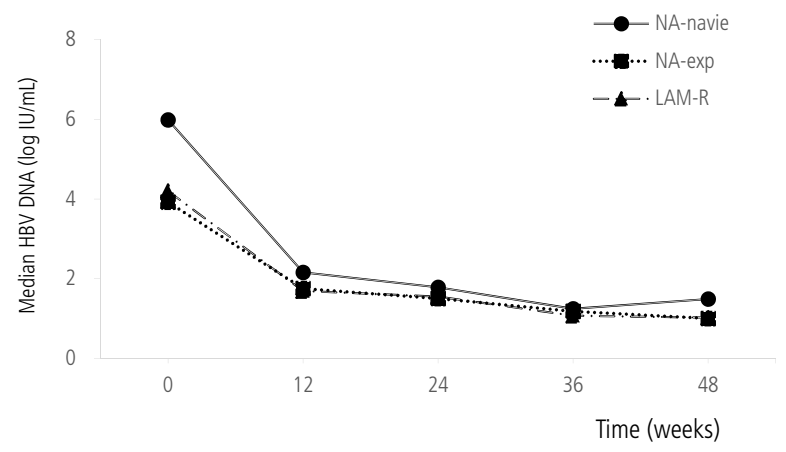

Figure 1. Mean changes in serum HBV DNA level during TDF treatment. $H B V$, hepatitis B virus; NA, nucleos(t)ide analogue; LAM- $R$, lamivudine resistance.

DNA in comparison of CVR between NA-naïve and NA-exp group, we analyzed CVR between two groups according to baseline serum HBV DNA level. For patients with baseline serum HBV DNA $<4.3 \log \mathrm{IU} / \mathrm{mL}, \mathrm{CVR}$ in NA-naïve patients $(\mathrm{n}=13,92.3 \%)$ was not significantly different compared with NA-exp group ( $n=43,82.7 \%)$, or LMV-R group ( $n=15,92 \%$ ) (Fig. 3A). For patients baseline serum HBV DNA $\geq 4.3 \log \mathrm{IU} / \mathrm{mL}$, CVR in NA-naïve group ( $n=49,65.2 \%$ ) had tendency to be higher compared with NA-exp group ( $n=31$, $50.3 \%, P=0.117$ ), or LMV-R group ( $\mathrm{n}=16,43 \%, P=0.063$ ) (Fig. 3B).

ALT normalization at week 48 was achieved in $82.6 \%$ (Fig. 4). Proportion of ALT normalization were also not significantly different compared NA-naive (79.3\%) with NA-Exp (88.9\%) or LAM-R (89.7\%) group.

HBeAg loss occurred in 9.6\% (9/94). HBeAg loss was $5.0 \%$ in NA-naive patients, $13.0 \%$ in NA -exp group and $12.0 \%$ in LAM-R group respectively. Virological breakthrough developed in $5 \mathrm{pa}-$ 



Figure 2. Cumulative probability of CVR to TDF according to variables. (A) omparison of CVR rate according to NA-experience. CVR rate in NAnaïve group was not significantly different from that in NA-exp group or LAM-R group. (B) Comparison of CVR rate according to HBeAg status. CVR rate was significantly higher in $\mathrm{HBeAg}$ negative patients than $\mathrm{HBeAg}$ positive patients. (C) Comparison of CVR rate according to baseline serum HBV DNA. CVR rate was significantly higher in patients with serum HBV DNA <4.3 log IU/mL than those with serum HBV DNA $\geq 4.3 \log \mathrm{IU} /$ $\mathrm{mL}$. CVR, complete virological response; TDF, tenofovir disoproxil fumarate; $N A$, nucleos(t)ide analogue; LAM- $R$, lamivudine resistance.
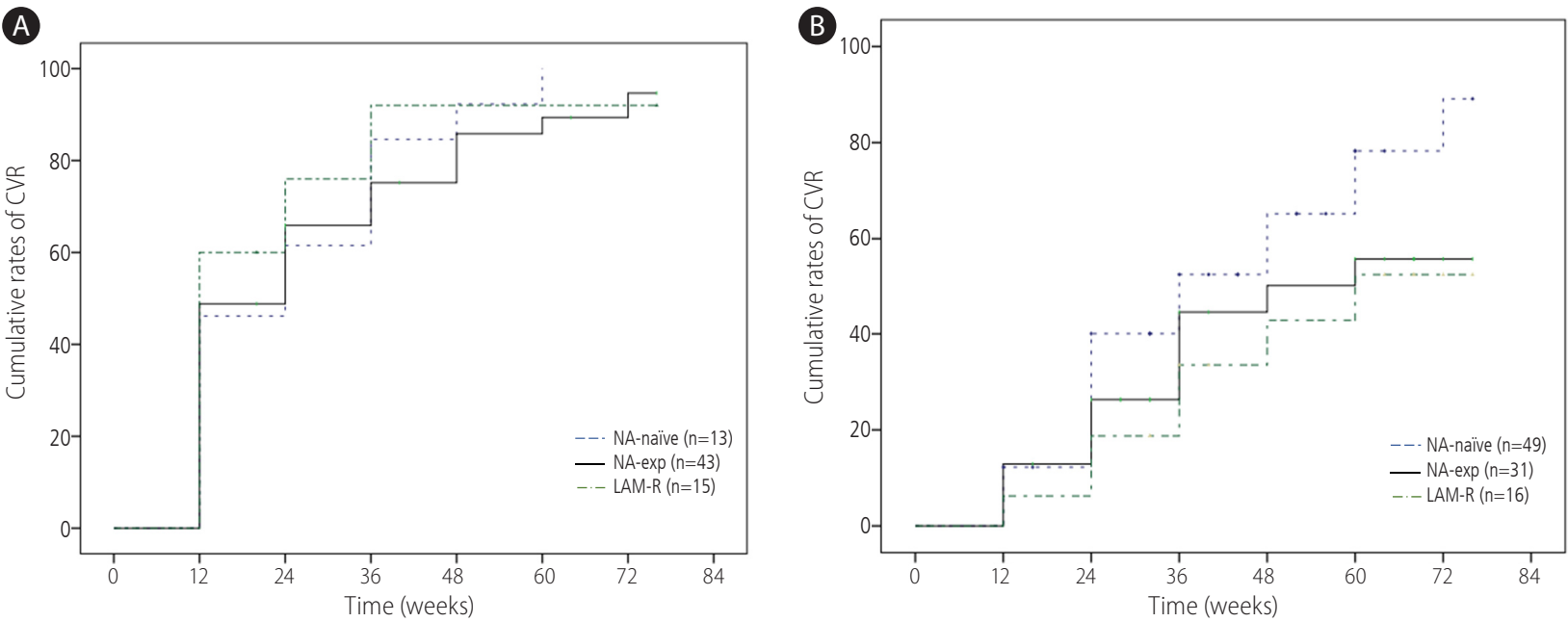

Figure 3. Cumulative probability of a CVR to TDF according to baseline serum HBV DNA. (A) CVR in patents with baseline serum HBV DNA <4.3 log IU/mL. CVR in NA-naïve patients ( $n=13,92.3 \%$ ) was not significantly different from that in NA-exp group ( $n=43,85.8 \%)$, or LMV-R group ( $n=15,92 \%)$. (B) CVR in patents with baseline serum HBV DNA $\geq 4.3 \mathrm{log} \mathrm{IU} / \mathrm{mL}, C V R$ in NA-naïve patients ( $n=49,65.2 \%$ ) had tendency to be higher as compared with NA-exp group ( $n=31,50.3 \%$. $P=0.117$ ), or $L M V-R$ group $(n=16,43 \%, P=0.063)$. CVR, complete virological response; TDF, tenofovir disoproxil fumarate; NA, nucleos(t)ide analogue; LAM-R, lamivudine resistance. 


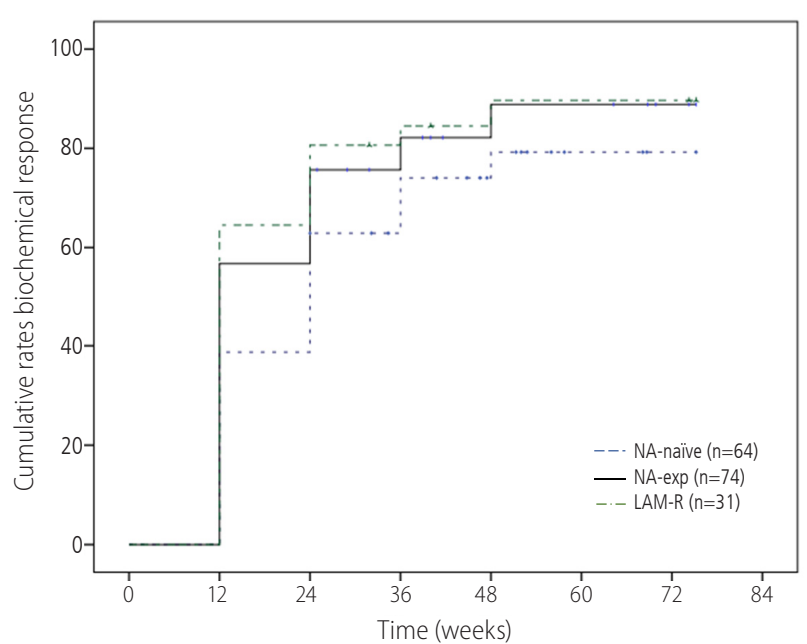

Figure 4. Cumulative probability of a biochemical response to TDF monotherapy. The biochemical response rate did not differ significantly between the NA-naïve group and the NA-exp group or the LAM-R group. TDF, tenofovir disoproxil fumarate; NA, nucleos(t)ide analogue; LAM- $R$, lamivudine resistance.

tients but no genotypic resistance was identified. Serum HBV DNA declined without medication change in four patients except one patient who was lost to follow up.

\section{Predictive factors for a complete virological response to TDF monotherapy}

Univariate Cox regression analyses of the total patients revealed that baseline serum HBV DNA level (HR, 0.830; $95 \% \mathrm{Cl} 0.888$ to $0.911)$ and $\mathrm{HBeAg}$ negativity ( $\mathrm{HR}, 1.685 ; 95 \% \mathrm{Cl} 1.096$ to 2.590$)$ were significantly associated with a CVR. Age, gender, baseline ALT, liver cirrhosis, NA-experience and resistance to lamivudine did not show any association with a CVR. In multivariate Cox proportional hazard model including variables with $P$-value of $\leq 0.1$ and NA-experience which might be a clinically significant factor, baseline serum HBV DNA was the only predictive factor for a CVR (HR, 0.809; 95\% Cl 0.729-0.898).

\section{Safety}

There were no case who discontinued TDF for significant adverse events during the TDF treatment. Renal function was maintained well in all patients. Also there was no patient with hypophosphatemia during the TDF treatment.

\section{DISCUSSION}

This study evaluated the therapeutic efficacy of TDF monotherapy both in NA-naïve and NA-experienced patients, and revealed that TDF monotherapy was highly effective in NA-experienced and LAM-resistant patients as well as NA-naïve patients. Serum HBV DNA reduction seemed to be greater in NA-naïve patients than NA-experienced patients in this study. However these differences of HBV DNA changes might be attributed to lower baseline serum HBV DNA in NA-experienced patients who had been closely monitored and had chance to take TDF in a state of relatively low serum HBV DNA. Actually, serum HBV DNA reduction in NA-experienced patients with similar baseline serum HBV DNA with NAnaiive patients was not different in that in with NA-naive patients. CVR rate and time to CVR were similar between NA-naïve and NA-Exp or LAM-R groups. However, in subgroup analysis for patients with baseline serum HBV DNA $\geq 20,000 \mathrm{IU} / \mathrm{mL}$ to compensate the difference of baseline serum HBV DNA which is the most important predictive factor for CVR, CVR tended to be lower in NA-exp or NA-. This study also investigated the predictive factors for CVR using Cox regression analysis. In univariate analysis, baseline serum HBV DNA and HBeAg were predictive factors for CVR. In multivariate analysis, only baseline serum HBV DNA was an independent predictive factor for CVR. NA-experience and LAM-resistance were not significantly associated with CVR.

TDF monotherapy is highly effective and safe for long-term suppression of HBV in NA-naïve patients. ${ }^{8,9}$ For patients with NA resistance, combination therapy used to be a standard of care for the concern of multidrug resistance. However, several studies reported the efficacy of TDF monotherapy in NA-experienced patients was not different from that in NA-naïve patients. ${ }^{10,16}$ In addition, the efficacy of TDF monotherapy was comparable to that of TDF/emtricitibine and TDF/LMV combination therapy in LAM-resistant patients. ${ }^{17,18}$ There has been no report on TDF resistance in NA-experienced patients yet. Based on these studies, TDF monotherapy was currently recommend for LAM-resistant patients. ${ }^{19}$

In Korea, we had relatively short clinical experience of TDF therapy for CHB treatment since TDF was licensed in December 2012. TDF was reported to be effective (CVR rate at $1 \mathrm{yr} 85.0 \%$ ) and safe in NA-naïve Korean patients. ${ }^{14}$ There are also a few studies on the efficacy of TDF monotherapy in NA-experienced patients in Korea. Choi et al. reported the efficacy of TDF monotherapy was comparable to that of TDF and NA combination in patients with genotypic mutation. ${ }^{20}$ Lee et al also reported the comparison of TDF monotherapy and TDF/LMV combination. ${ }^{21}$ However, these 
studies included relatively small numbers of patients, and moreover there have been few studies on head-to-head comparison of the efficacy of TDF monotherapy between NA-naiive and NA-experienced patients in Korea and to address whether the extent of viral suppression is actually not different depending on the prior exposure to NA or antiviral resistance. The results of this study which revealed the similar antiviral efficacy of TDF monotherapy irrespective of NA-experience was consistent with previous studies. ${ }^{17,18} \mathrm{NA}$-experienced patients in this study had characteristically low baseline serum HBV DNA, which could raise CVR rate and be a confounder in interpreting the effect of NA-experience on CVR. Even after adjusting baseline serum HBV DNA though multivariate analysis, NA-experience was not associated with CVR. However, in subgroups with serum HBV DNA $\geq 20,000 \mathrm{IU} / \mathrm{mL}$, CVR tended to be lower in NA-experienced or LMV-resistant patients compared with NA-naïve patients.

Viral breakthrough developed in 5 NA-experienced patients who all had poor compliance to medication. No genotypical resistance to TDF was found and HBV DNA declined without medication change in these four patients except one who was lost to follow up. This finding suggested non-adherence to medication was an important cause of viral breakthrough in real clinical setting.

This study was limited by retrospective design, relatively small number of subjects and limited duration of follow-up. Therefore, long-term study for more subjects is mandatory to elucidate the long-term clinical and virological effects of TDF monotherapy in NA-experienced and LAM-resistant patients.

In conclusion, TDF monotherapy was effective for CHB treatment irrespective of prior NA experience or LAM resistance. However, for patients with high serum HBV DNA, CVR in NA-exp or LAM-R group tended to be lower than that in NA-naive group. Baseline serum HBV DNA was the only independent predictive factor for CVR.

\section{Conflicts of Interest}

No potential conflict of interest relevant to this article is reported.

\section{REFERENCES}

1. Liaw YF, Chu CM. Hepatitis B virus infection. Lancet 2009;373:582592.

2. Chen CJ, Yang HI, Su J, Jen CL, You SL, Lu SN, et al. Risk of hepatocellular carcinoma across a biological gradient of serum hepatitis B virus DNA level. JAMA 2006;295:65-73.
3. Iloeje UH, Yang HI, Su J, Jen CL, You SL, Chen CJ. Predicting cirrhosis risk based on the level of circulating hepatitis B viral load. Gastroenterology 2006;130:678-686.

4. Mommeja-Marin H, Mondou E, Blum MR, Rousseau F. Serum HBV DNA as a marker of efficacy during therapy for chronic HBV infection: analysis and review of the literature. Hepatology 2003;37:1309-1319.

5. Liaw YF. Impact of therapy on the long-term outcome of chronic hepatitis B. Clin Liver Dis 2013;17:413-423.

6. Woo G, Tomlinson G, Nishikawa Y, Kowgier M, Sherman M, Wong DK, et al. Tenofovir and entecavir are the most effective antiviral agents for chronic hepatitis B: a systematic review and Bayesian meta-analyses. Gastroenterology 2010;139:1218-1229.

7. Marcellin P, Heathcote EJ, Buti M, Gane E, de Man RA, Krastev Z, et al. Tenofovir disoproxil fumarate versus adefovir dipivoxil for chronic hepatitis B. N Engl J Med 2008;359:2442-2455.

8. Marcellin P, Gane E, Buti M, Afdhal N, Sievert W, Jacobson IM, et al. Regression of cirrhosis during treatment with tenofovir disoproxil fumarate for chronic hepatitis B: a 5-year open-label follow-up study. Lancet 2013;381:468-475.

9. Kitrinos KM, Corsa A, Liu Y, Flaherty J, Snow-Lampart A, Marcellin P, et al. No detectable resistance to tenofovir disoproxil fumarate after 6 years of therapy in patients with chronic hepatitis B. Hepatology 2014:59:434-442.

10. van Bommel F, de Man RA, Wedemeyer H, Deterding K, Petersen J, Buggisch $P$, et al. Long-term efficacy of tenofovir monotherapy for hepatitis B virus-monoinfected patients after failure of nucleoside/ nucleotide analogues. Hepatology 2010;51:73-80.

11. Patterson SJ, George J, Strasser SI, Lee AU, Sievert W, Nicoll AJ, et al. Tenofovir disoproxil fumarate rescue therapy following failure of both lamivudine and adefovir dipivoxil in chronic hepatitis B. Gut 2011;60:247-254.

12. Fung S, Gordon SC, Krastev Z, Horban A, Petersen J, Sperl J, et al. Tenofovir disoproxil fumarate in Asian or Pacific Islander chronic hepatitis B patients with high viral load (>l= 9 log copies/ml). Liver Int 2014.

13. Song BC, Cui XJ, Kim H. Hepatitis B virus genotypes in Korea: an endemic area of hepatitis B virus infection. Intervirology 2005;48:133137.

14. Ahn SS, Chon YE, Kim BK, Kim SU, Kim do Y, Ahn SH, et al. Tenofovir disoproxil fumarate monotherapy for nucleos(t)ide-naive chronic hepatitis B patients in Korea: data from the clinical practice setting in a single-center cohort. Clin Mol Hepatol 2014;20:261-266.

15. EASL Clinical Practice Guidelines: management of hepatitis $C$ virus infection. J Hepatol 2011;55:245-264.

16. van Bommel F, Zollner B, Sarrazin C, Spengler U, Huppe D, Moller B, et al. Tenofovir for patients with lamivudine-resistant hepatitis $B$ virus (HBV) infection and high HBV DNA level during adefovir therapy. Hepatology 2006;44:318-325.

17. Berg T, Marcellin P, Zoulim F, Moller B, Trinh H, Chan S, et al. Tenofovir 
is effective alone or with emtricitabine in adefovir-treated patients with chronic-hepatitis B virus infection. Gastroenterology 2010;139:12071217.

18. Baran B, Soyer OM, Ormeci AC, Gokturk S, Evirgen S, Bozbey HU, et al. Efficacy of tenofovir in patients with Lamivudine failure is not different from that in nucleoside/nucleotide analogue-naive patients with chronic hepatitis B. Antimicrob Agents Chemother 2013;57:1790-1796.

19. EASL clinical practice guidelines: Management of chronic hepatitis $B$ virus infection. J Hepatol 2012;57:167-185.

20. Choi K, Lee HM, Jun BG, Lee SH, Kim HS, Kim SG, et al. Efficacy of Tenofovir-based Rescue Therapy for Patients with Drug-resistant Chronic Hepatitis B. Korean J Gastroenterol 2015;65:35-42.

21. Lee Cl, Kwon SY, Kim JH, Choe WH, Lee CH, Yoon EL, et al. Efficacy and safety of tenofovir-based rescue therapy for chronic hepatitis B patients with previous nucleo(s/t)ide treatment failure. Gut Liver 2014;8:64-69. 